\title{
Melanosis oris with Poliosis
}

\author{
Deepa Benni ${ }^{1}$, Dhirendra Sirur², Ghousia Begum³ ${ }^{3}$ Satyajith Naik ${ }^{4}$
}

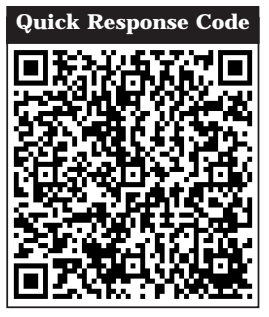

doi : 10.5866/2014.631625

${ }^{1}$ Maratha Mandals Institue of Dental Sciences and Research Centre, Belgaum

${ }^{2}$ Assistant Professor, Department of Oral Pathology, SDM College of Dental Sciences Dharwad

${ }^{3}$ Assistant Professor, Department of Pedodontics and Preventive Dentistry, Maruti Dental College, Bangalore. 4Professor, Department of Pedodontics and Preventive Dentistry, College of Dental Sciences, Davangere.

\section{Article Info:}

Received: April 15, 2014

Review Completed: May 12, 2014

Accepted: J une 13, 2014

Available Online: October, 2014 (www.nacd.in)

(C) NAD, 2014 - All rights reserved

Email for correspondence:

deepbenni@yahoo.co.in

\begin{abstract}
:
Pigmented lesions commonly seen in the oral cavity represent a variety of clinical entities, ranging from normal physiologic changes to clinical manifestations of systemic illnesses and malignant neoplasms. In this article, we are reporting a rare case of diffuse oral pigmented lesion throughout the oral mucosa with poliosis in a young child.
\end{abstract}

Key words: Pigmented lesion, Melanosis oris, Peutz J eghers syndrome.

\section{Introduction}

Pigmented lesions are more commonly seen in the oral cavity. These lesions represent a variety of clinical entities, ranging from physiologic changes (e.g., racial pigmentation) to manifestations of systemic illnesses (e.g., Addison's disease) and malignant neoplasms (e.g., melanoma and Kaposi's sarcoma). Pigmented lesion in the oral cavity can be a manifestation of systemic diseases. Therefore, an understanding of the etiology of various mucosal pigmentations seen in the oral cavity and appropriate evaluation of the patient are essential. ${ }^{1}$ A full medical and dental history, extraoral and intraoral examinations, and laboratory tests are required for evaluation of a patient presenting with pigmented lesion. ${ }^{2}$

Oral pigmentation may be endogenous or exogenous in origin. Melanin, hemoglobin, hemosiderin and carotene constitute endogenous pigments. Melanin pigmentation is most commonly and widely seen physiologic pigmentation of oral mucosa. Melanocytes in the basal layer of the epithelium produce melanin, which is transferred to adjacent keratinocytes via membrane-bound organelles called melanosomes. Melanin is also synthesized by nevus cells derived from the neural crest and can befound in the skin and mucosa. These melanin pigmented lesions may be brown, blue, grey or black, depending on the amount and location of melanin in the tissues. Exogenous pigmentation is commonly due to implantation of foreign-body in the oral mucosa. ${ }^{1}$

Pigmented lesion can befocal or diffuse/bilateral with early or adult onset. Diagnosis of such

\section{Indian Journal of Dental Advancements Journal homepage: www. nacd. in}


pigmented lesion should be made based on clinical and laboratory findings. In this paper we present the case of a $4 \mathrm{yr}$ old boy who had diffuse melanosis oris with Poliosis.

\section{Case Report}

A 4yr old male patient of I ndian origin reported to Department of Pedodontics and Preventive Dentistry, College of Dental Sciences, Davangere to undergo routine dental check up. Clinical examination of the oral cavity revealed generalized diffuse brownish to blackish pigmentation involving commissures of lip, labial mucosa, buccal mucosa, gingiva and hard palate (Figure 1-3). It was not associated with pain or any other signs of inflammation. Dental problems included initial caries lesion with 52, 51, 61 and 62. Extra oral examination revealed poliosis in left temporal region (Figure 4).

History revealed pigmentation to be present since birth with no variation in the intensity of pigmentation. There was no associated signs and symptoms, skin hyper-pigmentation. The patient was healthy and was not taking any medications. The family history did not reveal any such occurrences.

The differential diagnosis of diffuse pigmention in the oral mucosa of a $4 y r$ old child included racial (physiologic) pigmentation and Peutz jeghers syndrome. The patient was referred to pediatrician to rule out Peutz jeghers syndrome. The child underwent medical examination and ultrasound of abdomen. Abdominal ultrasound showed no polyposis. The child did not present any other associated features of Peutz jeghers syndrome.

Dental treatment was carried out and no medication was prescribed for pigmentation as it was considered most likely as physiologic pigmentation. The patient was followed up after 1 year to see any changes in the degree of oral mucosal pigmentation. On intra-oral examination no changes could be seen (Figure 5-A, B, C). The patient was scheduled for recall check up and has to be followed up medically if any signs of Peutz jeghers syndrome develop later in life.

\section{Discussion}

Despite the fact that oral mucosa has the same density of melanocytes as that of skin, but the oral mucosa rarely appears to be pigmented. Occasionally however patients, may show a focal area of melanin deposition as a response to local chronic conditions (mechanical trauma, tobacco smoking, chronic autoimmune mucositis), racial background (the darker a person's skin color the more likely they are to have oral pigmentation), or systemic medications, especially prolonged chloroquine intake. Also certain syndromes and systemic diseases may have oral pigmentation as part of their spectrum.

It is a challenge to diagnose pigmented lesions of the oral cavity and perioral tissues. Clinicians should evaluate and diagnose all alterations in pigment. But definitive diagnosis usually requires histopathol ogical evaluation. On the basis of history, clinical examination and laboratory investigations, there is an algorithm suggested by Kauzman A et al to guide the assessment of pigmented lesions of the oral cavity (Table 1). ${ }^{3}$

Early onset pigmentation includes physiologic pigmentation and pigmentation seen in Peutz J eghers syndrome. Physiologic pigmentation, which is common in African, Asian and Mediterranean populations, is due to melanocyte activity to a greater extent rather than a greater number of melanocytes. ${ }^{1}$ Physiologic pigmentation develops during the first two decades of life but may not be noticed by the patient until later. The col our ranges from light to dark brown. The most common intraoral site of such pigmentation is attached gingiva, where it appears as a bilateral, welldemarcated, ribbon-like, dark brown band usually sparing the marginal gingiva. ${ }^{4}$ Pigmentation of the buccal mucosa, hard palate, lips and tongue may also be pigmented which show as brown patches with poorly-defined borders. This pigmentation is asymptomatic and no treatment is required.

Peutz-J eghers syndrome is a rare genetic disorder which is associated with mutation of the LKBl gene on chromosome 19. ${ }^{5}$ The clinical features being pigmented mucocutaneous lesions, intestinal hamartomatous polyposis and a increased risk of cancer in many organs, including the small intestine, colon, stomach, pancreas, breast and genital tract. ${ }^{6}$ The melanotic spots of Peutz-J eghers syndrome are characteristically small and multiple, and are very distinct around the lips. Pigmented spots may also be seen in the oral mucosa, mucosa of the nose, conjunctiva and rectum, and on the skin of the extremities. ${ }^{7}$ As the melanotic spots are not associated with increased risk of melanoma they do not require any treatment. However, but the patient 


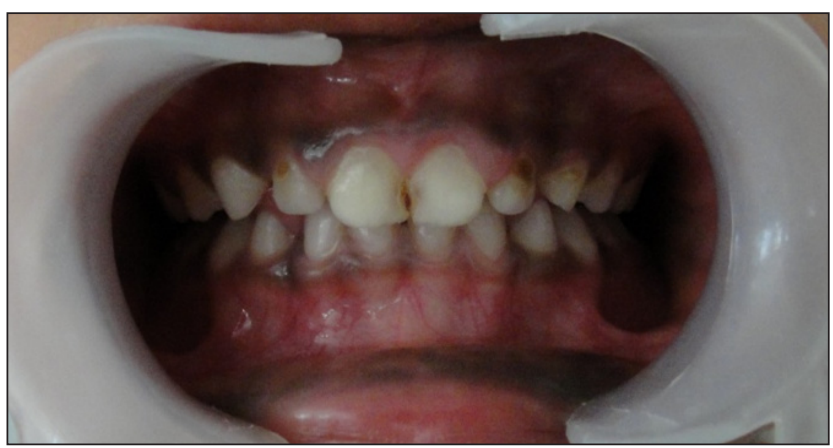

Figure 1: Intra Oral Photograph of 4 Year old patient

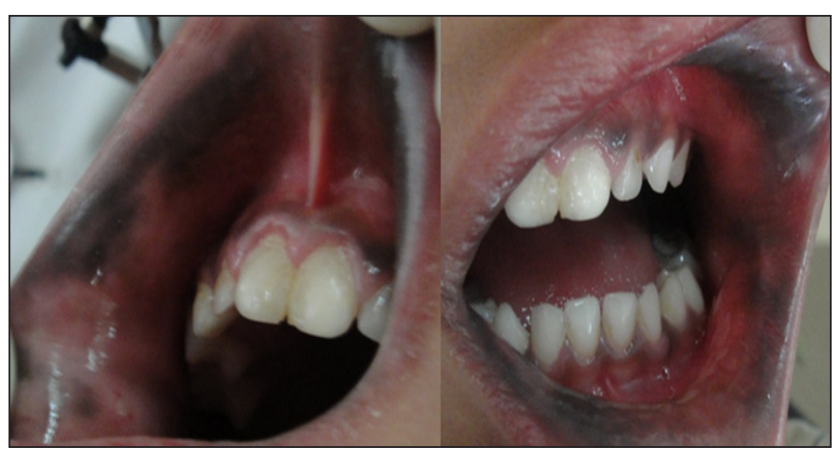

Figure 2: Intra Oral Photograph-Left and Right buccal mucosa

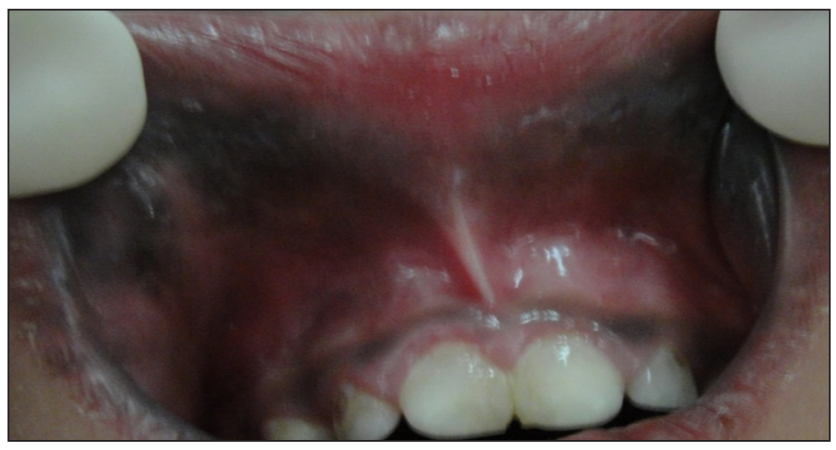

Figure 3: Intra Oral Photograph-Labial mucosa

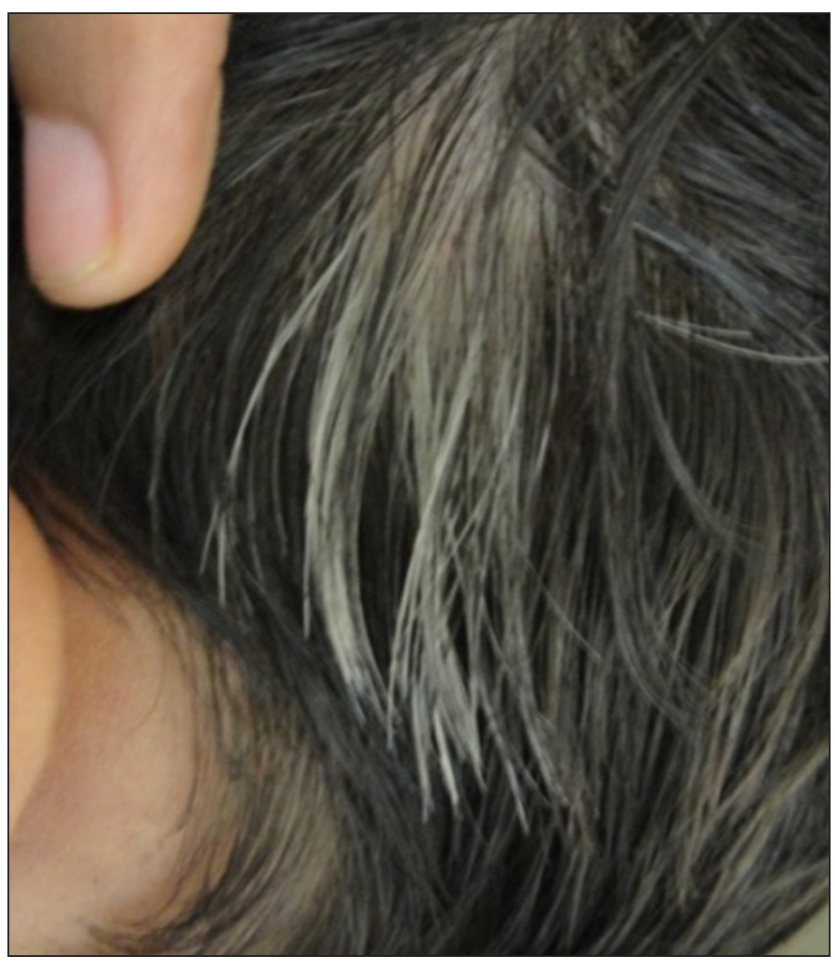

Figure 4: Poliosis in left temporal region

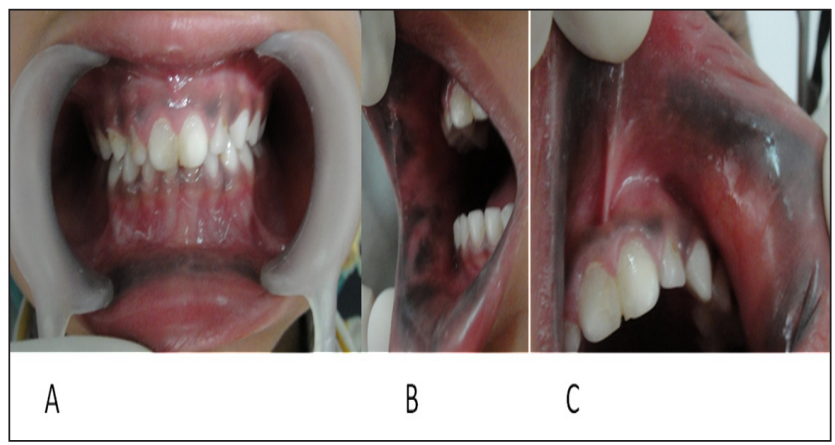

Figure 5: A, B, C lyear Follow up Photograph. A - Labial mucosa, gingiva. B - Right Labial and buccal mucosa. C - Left Labial mucosa.

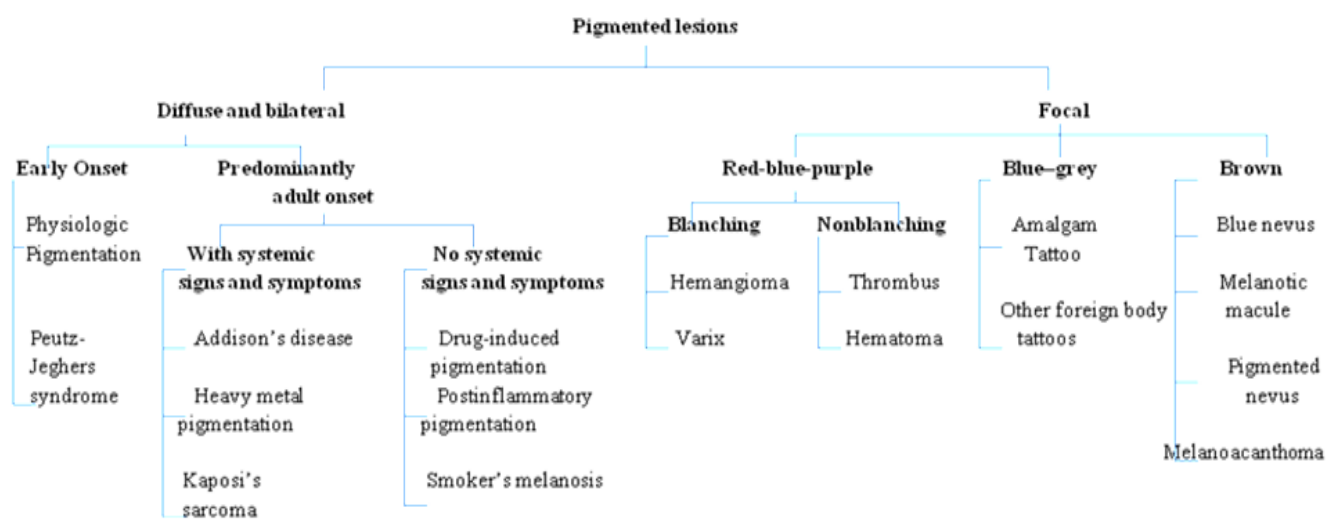

Table I: An algorithm for evaluation of pigmented lesions of the oral cavity. 
has be monitored for the development of internal malignancies or any complications.

Polyposis in this syndrome occurs chiefly in the jejunal part of the small intestine, but also throughout the whole gastro-intestinal tract including the rectum and very often the stomach, the latter accounting for haematemesis as a rare presenting symptom. Polyps haveal so been recorded in the nose and bladder. ${ }^{8}$ The median time for the first presentation of polyps is about 11-13 years of age, and by the age of 20 years approximately $50 \%$ will have experienced symptoms.

Polyps can occur without pigmentation, just as pigmentation can occur without symptoms or evidence of polyposis, but these cases may not belong to this syndrome. ${ }^{8}$ Hyperpigmentations can even disappear during adolescence. Diagnosis is defined by the presence of histopathologically confirmed hamartomatous polyps and at least two of the following clinical criteria: family history, hyperpigmentation and polyps in the small bowel.9,10

Poliosis is the term given to signify a localized patch of white hair. Most frequently, poliosis presents as a white forelock, but it can involve a patch of white hair in normally pigmented hair anywhere on the body. Poliosis can occur in healthy people and may represent no more than an anomaly of hair and skin pigmentation. However, poliosis is also observed in association with a wide variety of conditions. In its mildest form, a minor genetic defect called piebaldism may have poliosis as one of the symptom. Somewhat more significant, poliosis may be associated with pigmentary disturbances of the eye, as well as hair loss in hypogonadism and thyroid diseases. Poliosis may be a result of localized changes in the skin. Patches of white hair growth in the areas of affected skin may be seen in various kinds of nevi and focal skin cancer.

Oral melanotic pigmentation has to be differentiated from certain other similar conditions exhibiting hyperpigmentation. The two most important causes of post inflammatory hyper pigmentation are lichen planus and lupus erythematosis. There are certain endocrinal hyper pigmentations. Addison's disease causes a darkening of the oral mucosa which is irregular, patchy and found on the gums. Metal deposition can cause discolouration either from copper as in Wilson's disease or from amalgam as in an amalgam tattoo. It could be associated with syndromes as in Leopard syndrome where pigmentation is seen all over the body. Antimalarial drugs like chloroquine can also cause muscosal hyperpigmentation which also occurs on other body parts like the shins.
There are no cases reported in the literature with the melanosis oris in a child to the extent that is reported in this case. This case is a rare case of melanosis oris with Poliosis which has to befollowed up regularly to see any changes in the pigmentation if it occurs.

\section{Conclusion}

Pigmented lesions of the oral mucosa range from the extremely common and harmless (eg, physiologic pigmentation, amalgam tattoo) to the rare and deadly (eg, malignant melanoma). Various pigmented lesions can have similar clinical presentations, posing a diagnostic dilemma for the dentist. In the present paper, we have reported a case of melanosis oris with poliosis which is not so common in children.

This case report adds to the literature the importance of detailed clinical examination of the oral and oropharyngeal mucosa for the proper diagnosis of pigmentation disorders. A high level of suspicion with careful history and a thorough physical examination including the oral cavity and the neck should be performed by involved health providers like general practitioners, dentists. Minimal, atypical signs or slight deviation from the normal must be seriously considered.

\section{References}

1. Eisen D. Disorders of pigmentation in the oral cavity. Clin Dermatol 2000; 18(5):579-587.

2. Kleinegger $\mathrm{CL}, \mathrm{H}$ ammond $\mathrm{HL}$, Finkelstein $\mathrm{MW}$. Oral mucosal hyperpigmentation secondary to antimalarial drug therapy. Oral Surg Oral Med Oral Pathol Oral Radiol Endod 2000; 90(2):189-194.

3. Kauzman A, Pavone M, Blanas N, Bradley G. Pigmented lesions of the oral cavity: review,differential diagnosis, and case presentations. J Can Dent Assoc. 2004; 70(10): 682-683.

4. Gaeta GM, Satriano RA, Baroni A. Oral pigmented lesions. Clin Dermatol 2002; 20(3): 286-288.

5. Hemminki A, Markie D, Tomlinson I, Avizienyte E, Roth S, Loukola A, and others. A serine/threonine kinase gene defective in Peutz-J eghers syndrome. Nature 1998; 391(6663):184-187.

6. Hemminki A. Themolecular basis and dinical aspects of PeutzJ eghers syndrome. Cell Mol Life Sci 1999; 55(5):735-750.

7. McGrath DR, Spigel man AD. Preventive measures in PeutzJ eghers syndrome. Fam Cancer 2001; 1(2):121-125.

8. Leslie G. Andrews. Intestinal Polyposis Associated With Melanosis Oris. Archives of Disease in childhood 1954; 455456.

9. Chen HM, Fang J Y. Genetics of the hamartomatous polyposis syndromes: a molecular review. Int J Colorectal Dis 2009; 24: 865-874.

10. Marcela Kopacova, Ilja Tacheci, Stanislav Rejchrt, J an Bures. Peutz-J eghers Syndrome. Diagnostic and therapeutic approach. World J Gastroenterol 2009; 15(43): 5397-5408. 